\title{
Localized Electronic Excitations in NiO Studied with Resonant Inelastic X-Ray Scattering at the Ni $M$ Threshold: Evidence of Spin Flip
}

\author{
S. G. Chiuzbăian, ${ }^{1, *}$ G. Ghiringhelli, ${ }^{2}$ C. Dallera, ${ }^{2}$ M. Grioni, ${ }^{3}$ P. Amann,,${ }^{1,}$ X. Wang, ${ }^{3}$ L. Braicovich, ${ }^{2}$ and L. Patthey ${ }^{1}$ \\ ${ }^{1}$ Paul Scherrer Institut, CH-5232 Villigen PSI, Switzerland \\ ${ }^{2}$ INFM-Dipartimento di Fisica, Politecnico di Milano, p. Leonardo da Vinci 32, 20133 Milano, Italy \\ ${ }^{3} I P N$, Ecole Polytechnique Fédérale, CH-1015 Lausanne, Switzerland \\ (Received 24 February 2005; published 31 October 2005)
}

\begin{abstract}
We studied the neutral electronic excitations of $\mathrm{NiO}$ localized at the $\mathrm{Ni}$ sites by measuring the resonant inelastic x-ray scattering (RIXS) spectra at the $\mathrm{Ni} M_{2,3}$ edges. The good energy resolution allows an unambiguous identification of several spectral features due to $d d$ excitations. The dependence of the RIXS spectra on the excitation energy gives evidence of local spin flip and yields a value of $125 \pm 15 \mathrm{meV}$ for the antiferromagnetic exchange interaction. Accurate crystal field parameters are also obtained.
\end{abstract}

PACS numbers: 78.70.Ck, 71.27.+a, 75.30.Et, 78.70.Dm

Determining the nature of the electronic states in strongly correlated materials is a prerequisite to understanding their rich and intriguing physical properties, including magnetism and high $T_{c}$ superconductivity. Photoemission (inverse photoemission) can directly probe the electron removal (addition) spectral function, but neutral excitations like the $d d$ excitations in the $3 d$ transition metal $(3 d \mathrm{TM})$ oxides and related materials require a different approach. Such atomiclike excitations, which correspond to a local rearrangement of the $3 d$ electrons, play a crucial role in many properties of these materials, and carry important information on magnetic interactions. They were traditionally the domain of optical absorption and electron energy loss experiments. More recently, however, it was shown that resonant inelastic x-ray scattering (RIXS) [1] also probes $d d$ excitations, with the distinctive advantages of a resonant spectroscopy although the resolution has been a limiting factor. Here we present RIXS data having good resolution and measured at the Ni $M$ edge $(3 p)$ of the benchmark compound $\mathrm{NiO}$, and identify magnetic excitations probed with RIXS in an independent and complementary way to neutron studies. In RIXS, the resonant absorption of a photon by a core electron leads to a radiative deexcitation which leaves the system either in its ground or in a neutral excited state [1,2]. Thus $d d$ excitations - including excitations of magnetic interestyield characteristic spectral losses dispersing with incident photon energy (Raman regime) and information is projected on the cation site $[3,4]$. In RIXS studies the $L$ edges have been more commonly employed mainly because the scattering cross section is much higher and the $2 p$ spinorbit splitting is larger than at $M$ edges. However, the instrumental linewidth is much broader at $L$ edges. Only recently Ghiringhelli et al. [5,6] have effectively used $L_{3}$ RIXS to study $d d$ excitations with an instrumental bandwidth considerably smaller than $1 \mathrm{eV}$. In contrast, $M$ RIXS, despite the first impressive demonstration by Kuiper et al. [7], was seldom used in recent years, and then only at the $\mathrm{Cu}$ edge to the authors' knowledge [8].
We have thus exploited an energy resolution down to $130 \mathrm{meV}$ in the range $65-72 \mathrm{eV}$ (RIXS at the $M$ edge of $\mathrm{Ni}$ ) to study the neutral local electronic excitations of $\mathrm{NiO}$. The interest of the study is not only in the paradigmatic importance of this compound and in the precise location of the spectral features allowed by the increased resolution, but in more fundamental aspects. In fact, we were able to address the local spin-flip excitations at the Ni-site in the final state of RIXS. By spin-flip excitations we refer here to transitions involving a change in the orientation of the local spin moment on Ni cation sites with respect to the orientation of spin moments on neighboring sites [9]. This idea, put forward by de Groot et al. [10] with a theoretical estimate for the $L$ edge, has never been implemented experimentally to the authors' knowledge, probably due to the difficulty of the experiment. We note that: (i) the spin-flip transitions cannot be observed in direct optical absorption within the $3 d$-manifold in the infrared and in the visible [11] where these transitions are strongly suppressed; (ii) the spin-flip transitions can indeed be seen in electron energy loss spectroscopy [12], basically in a surface sensitive mode; RIXS, even at low energies, is much more bulk sensitive so that it adds new relevant information; (iii) up to now, this information has been obtained indirectly by modelling the dispersion of spin waves [13]; the present paper gives a complementary approach to neutron spectroscopy.

Another result of the present work is the accurate determination of the crystal field parameters needed to describe the Ni-atom site. This is much easier in these accurate measurements than in cases having larger energy linewidths at the $L$ edges as in Refs. [14-16]. In the following, the crystal field parameters are the best values in a single ion model having octahedral symmetry without hybridization to the ligand and with a single configuration $\left(3 d^{8}\right.$ in this case). This is the traditional way of defining the parameters in the crystal field approach, as found commonly in the literature on $3 d \mathrm{TM}$ compounds. Thus, our values are directly comparable to the literature without the 
need of renormalizing for interaction with the surroundings of the ion. $\mathrm{NiO}$ has been considered to be prototypical among the strongly correlated systems for the difficulties involved in the understanding the nature of its gap $[17,18]$. Below $T_{N}=523 \mathrm{~K}, \mathrm{NiO}$ exhibits antiferromagnetic ordering with the spin moments along the $\langle 11 \overline{2}\rangle$ directions. The sample was a single crystal $\mathrm{NiO}(100)$ cleaved in air and oriented in a vertical plane at $25^{\circ}$ grazing incidence. The scattered photons were detected at $90^{\circ}$ from the incident beam. The measurements were carried out at the SIS beamline (Swiss Light Source) $[19,20]$ at room temperature and $2 \times 10^{-10}$ mbar base pressure, using linearly polarized light, with the polarization in the horizontal plane (resolving power $E / \Delta E \cong 3000$ for an exit slit opening of $200 \mu \mathrm{m})$. The scattered light was recorded with a Rowland-circle grazing incidence spectrograph [21] with a 300 lines $/ \mathrm{mm}$ grating ( $3 \mathrm{~m}$ Rowland radius) and entrance slit set to $30 \mu \mathrm{m}$. It took about 20-30 min or several hours to record a RIXS spectrum with the first and second diffraction order of the grating, respectively.

The soft x-ray absorption spectrum (XAS) at the $M$ threshold (Fig. 1) was recorded by monitoring the sample drain current and by normalizing to the reference current taken from a gold mesh. The measured XAS is in good agreement with the previously reported data $[22,23]$. The two main features with maxima around 66.7 and $70.0 \mathrm{eV}$ are atomic multiplet rather than spin-orbit split peaks [24].

The RIXS spectra measured with several excitation energies at the Ni $M$ edge (shown by bars in Fig. 1) are presented in Fig. 2 versus the energy transferred to the sample $h \nu_{\text {in }}-h \nu_{\text {out }}$ (combined resolution $200 \mathrm{meV}$ ) while Fig. 3 gives two spectra with higher resolution $(130 \mathrm{meV})$, obtained with the second order of the spectrograph past the sample. Before any interpretation the results deserve the following comments: (i) The spectra are dominated by the elastic peak (feature A) (see Figs. 2 and 3) and the inelastic features (B-D) are seen only by drastically expanding the spectra. This is analogous to what was seen by Kuiper et al. in $\mathrm{Sr}_{2} \mathrm{CuO}_{2} \mathrm{Cl}_{2}$ [7] and shows the need for a high-resolution and flux beamline. (ii) All inelastic peaks disperse with the

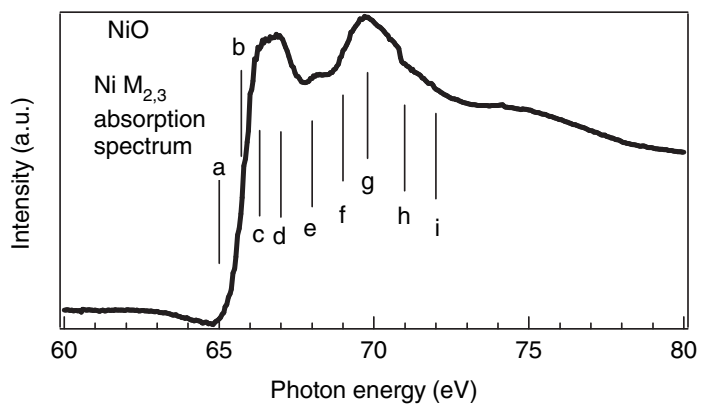

FIG. 1. Total electron yield XAS spectrum recorded across the $M$ threshold of $\mathrm{Ni}$ in NiO. The excitation energies used for the RIXS spectra are indicated with lines and denoted with small letters. excitation energy also well above the threshold. This is at variance with the behavior at the $L$ edge where RIXS shows also fluorescence and charge-transfer excitations when exciting a few $\mathrm{eV}$ above the threshold [14,15]. Thus, the extensive Raman behavior makes $M$ RIXS very useful to study localized excitations. (iii) The evolution of the relative spectral weights with the incident energy across the resonance shows that feature $\mathrm{B}$ dominates at lower energies while across the resonance the intensity of feature $\mathrm{C}$ becomes sizable. (iv) Fig. 2 shows that the center of gravity of feature B is slightly dependent on the excitation energy across the resonance; the maximum shift (about $60 \mathrm{meV}$ ) is reached with $66.3 \mathrm{eV}$ photons. (v) In the higher resolution measurements of Fig. 3, the feature $\mathrm{C}$ in the spectrum at $68.2 \mathrm{eV}$ is clearly not a single peak. In the expanded view of peak $\mathrm{C}$ in the inset of Fig. 3, this feature (red dots) is well fitted by two components with a separation of $170 \mathrm{meV}$ (the line shape is taken from the elastic peak, giving the instrumental response with a further Lorentzian broadening of $50 \mathrm{meV}$ ). The double nature of the peak appears at resonance and is not found below resonance, as shown by the spectrum with excitation $(b)$ (blue triangles).

As anticipated above, the spectral features are assigned with the use of a crystal field approach. The transferred energy corresponds to the excitation of the system in the final state. Thus the assignments simply require the comparison with the low energy excitations of the system. The positions of all peaks are well explained by the $d d$ excita-

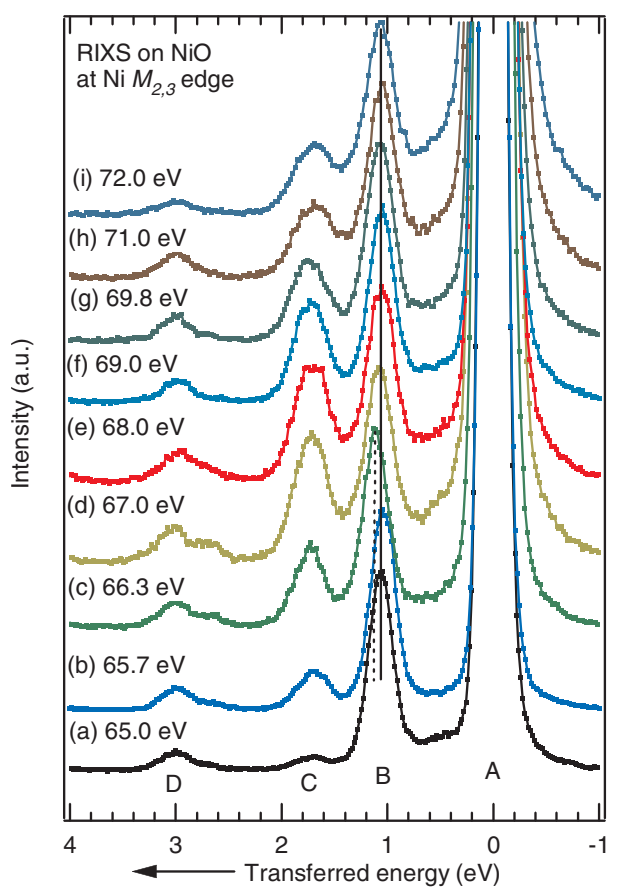

FIG. 2 (color). Energy-dependent RIXS spectra measured at the Ni $M_{2,3}$ resonances in $\mathrm{NiO}$. All spectra are normalized to the intensity of the first loss. 
transferred energy $(\mathrm{eV})$

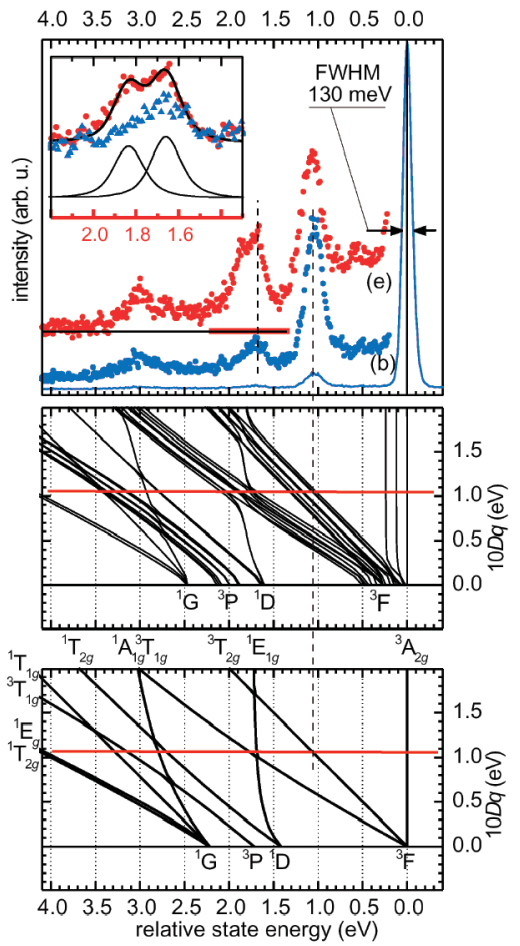

FIG. 3 (color). Upper panel: High energy resolution spectra taken with the second order of the spectrometer at the excitation energies $(b)$ and (e) (see Fig. 1). The inset gives an expansion of the spectra in the region marked by the red horizontal bar $[(e)$ in red dots and $(b)$ blue triangles]. The spectrum $(e)$ is decomposed in two components whose sum is the heavy black line. The two lower panels give the Sugano-Tanabe diagrams versus crystal field $10 D q$ (see text).

tions, confirming that we do not see charge-transfer excitations. We optimized $10 D q$ (which measures the energy distance between $e_{g}$ and $t_{2 g}$ states in octahedral symmetry) in the calculation of the $d d$ neutral excitations (final states in the scattering) with the COWAN code [25] plus a crystal field with a rescaling to $70 \%$ of the Slater integrals to account for intra-atomic correlation beyond the HartreeFock scheme. The results are collected versus $10 D q$ in the bottom of Fig. 3 (Sugano-Tanabe diagrams [26]) without (lower panel) and with spin-orbit and exchange $(120 \mathrm{meV}$, a reasonable value as shown below). The figures also give the symmetry labels. The optimal value obtained by comparing with the values without exchange is $10 D q=$ $1.05 \mathrm{eV}$. This is particularly clear from the first loss B corresponding to the $d d$ transition ${ }^{3} A_{2 \mathrm{~g}} \rightarrow{ }^{3} T_{2 \mathrm{~g}}$, at energy $10 D q$. With exchange the Sugano-Tanabe diagrams give a manifold of lines (see central panel of Fig. 3) in place of a single line, so that the spectra have a detailed structure giving information on the exchange. The spectral intensities of these components are determined by the selection rules in the scattering, by the transition matrix elements, and by the interference between scattering paths. The discussion of the detailed structure requires calculations of the spectral functions with the Kramers-Heisenberg formula [27].

An illuminating set of theoretical spectra at various excitation energies is given in Fig. 4 obtained with the above parameters and with an exchange of $120 \mathrm{meV}$, with lifetime broadening in the intermediate state of $1 \mathrm{eV}$ [28] and $50 \mathrm{meV}$ in the final state (without including the instrumental broadening, for clarity). The magnetization and the photon incidence are along the octahedral axis $\left(C_{4 \mathrm{~h}}\right.$ symmetry). The scattering geometry and the incident photon polarization are as in the experiment. The figure is the $M$ edge analogous to calculations by de Groot et al. for the $L_{3}$ edge [10]. The spectral functions are calculated either not including (red lines) or including (heavy black lines) the interatomic exchange interaction, in order to highlight the new structures related to the spin-flip excitations. These are satellites (marked by arrows in Fig. 4) at higher transferred energy due to the energy cost of local spin flip on the cation site. The satellites resonate at certain incident energies so that their effect is expected to be seen in the experiment as a modification of the spectra at increasing energy across the resonance. This is due to the fact that distinct incident energies select different intermediate states thus leading to different weights in the final states. The region A near the elastic peak (and looking very promising from Fig. 4) cannot be used because in the experiment this region is submerged by the tail of the huge elastic (Rayleigh) peak. A reliable decomposition of the measured spectra seems unrealistic. On the other hand, there are two pieces of evidence for spin flip discussed here in order of increasing importance: (i) In region $\mathrm{B}$ at resonance there is a clear spin-flip satellite basically separated by one exchange from the main peak. A simulation of the experiment, taking into account the instrumental linewidth, is shown in the inset of Fig. 4 giving

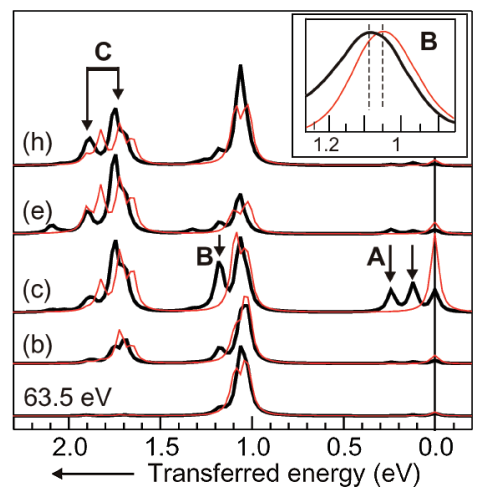

FIG. 4 (color). Theoretical RIXS spectra excited at the energies $63.5 \mathrm{eV},(b),(c),(e)$ and $(h)$ (see Fig. 1). The spectra without exchange interaction are given by the red line and those with exchange by the heavy black line. The inset gives the expected shape of feature B in case (c) by accounting for the experimental band-pass. 
an expanded view of region B. Although the resolving power is not sufficient to see the satellite, a clear shift of the peak at resonance is the consequence of the spin flip as is indeed the case in the experimental results. As stressed above, the experiment shows a shift across resonance (see Fig. 2), which is qualitative evidence for local spin flip, compatible with an exchange between about 80 and $150 \mathrm{meV}$. In the inset, the simulation takes approximately into account the multidomain structure of the sample with an average of spectra with different orientations between the $\mathrm{x}$ rays and the magnetization. (ii) More quantitative is the analysis of region $\mathrm{C}$ at resonance and above. In the theoretical spectra there are basically two main features whose separation is greater than the exchange and increases with the magnitude of the considered exchange. Thus it is easier to resolve experimentally the two peaks in region $\mathrm{C}$ than in the region $\mathrm{B}$. This is indeed the case and we have already shown that feature $\mathrm{C}$ in the high-resolution spectra has two components separated by $170 \mathrm{meV}$ (inset of Fig. 3). This separation is accurately fitted in the highresolution spectrum with excitation $e$. With the constraint of keeping the $10 D q$ value already obtained from the positions of the spectral features we derive an exchange of $125 \pm 15 \mathrm{meV}$ (the $120 \mathrm{meV}$ used in all preceding figures can hardly be distinguished from this value). The result is a rather direct measurement based on RIXS. Strong support for this analysis comes from the fact that in the experiment the satellite resonates and is basically suppressed below resonance, as shown by the inset of Fig. 3. This trend is also found in our calculations.

The exchange has been obtained in a scheme based on a pure crystal field without hybridization with the ligand band. The renormalization of the exchange due to this effect should be very small, making our estimate very reliable. In fact we use a separation between two nearby spectral features that would be renormalized basically in the same way, because both spins are present almost with the same weight in the ligand band. The present value compares well with the value of $114 \mathrm{meV}$ obtained from spin-wave analysis [13]. This demonstrates the consistency between the two approaches and shows that future highresolution RIXS will be very useful in the study of exchange effects. Note that this is a local probe not requiring macroscopic magnetization and also working in antiferromagnetic systems as in our case.

In conclusion, we have presented RIXS measurements at the $M$ edge of $\mathrm{Ni}$ in $\mathrm{NiO}$, which shows that high quality data can now be obtained despite the very low cross section. This not only allows a precise location of the spectral features and the accurate tuning of the parameters in theoretical models, but also gives direct experimental access to exchange effects. The present approach gives a cross fertilization with the work based on neutron scattering.
The work was performed at the Swiss Light Source, Paul Scherrer Institut, Villigen, Switzerland. The technical support provided by J. Krempaski and F. Dubi is gratefully acknowledged. L. B., C.D., and G.G. are grateful to R. Gusmeroli and to F. Fracassi for the help with the computational part.

*Electronic address: gheorghe.chiuzbaian@psi.ch ${ }^{\dagger}$ Currently at Institut für Experimentalphysik, Leopold Franzens Universität, 6020-Innsbruck, Austria.

[1] A. Kotani and S. Shin, Rev. Mod. Phys. 73, 203 (2001).

[2] J.-E. Rubensson et al., Appl. Phys. A 65, 91 (1997).

[3] S. Tanaka and A. Kotani, J. Phys. Soc. Jpn. 62, 464 (1993).

[4] S. M. Butorin et al., Phys. Rev. B 54, 4405 (1996).

[5] G. Ghiringhelli et al., Phys. Rev. Lett. 92, 117406 (2004).

[6] See also the report of a study at $\mathrm{Mn} L$ edges with $0.3-0.35 \mathrm{eV}$ combined experimental linewidth, at URL http://www.esrf.fr/NewsAndEvents/Spotlight/ spotlight9SoftRIXS.

[7] P. Kuiper et al., Phys. Rev. Lett. 80, 5204 (1998).

[8] L.-C. Duda et al., Surf. Rev. Lett. 9, 1103 (2002).

[9] To be distinguished from intra-atomic spin-flip transitions which account for the flip of a single electron spin and imply a change in multiplicity (see for example J.-J. Gallet et al., Phys. Rev. B 54, R14238 (1996).)

[10] F. M. F. de Groot et al., Phys. Rev. B 57, 14584 (1998).

[11] M. Fiebig et al., Phys. Rev. Lett. 87, 137202 (2001).

[12] B. Fromme, $d$-d Excitations in Transition Metal Oxides (Springer Verlag, Berlin, 2001), and the references therein.

[13] M. Hutchings and E. Samuelsen, Phys. Rev. B 6, 3447 (1972).

[14] H. Ishii et al., J. Phys. Soc. Jpn. 70, 1813 (2001).

[15] M. Magnuson et al., J. Phys. Condens. Matter 14, 3669 (2002).

[16] G. Ghiringhelli et al. (unpublished).

[17] G. Ghiringhelli et al., J. Phys. Condens. Matter 17, 5397 (2005).

[18] O. Tjernberg et al., Phys. Rev. B 53, 10372 (1996).

[19] T. Schmidt et al., Nucl. Instrum. Methods Phys. Res., Sect. A 467, 126 (2001).

[20] U. Flechsig et al., Nucl. Instrum. Methods Phys. Res., Sect. A 467, 479 (2001).

[21] J. Nordgren and R. Nyholm, Nucl. Instrum. Methods Phys. Res., Sect. A 246, 242 (1986); we used the commercial model XES-350 produced by Gammadata (Sweden).

[22] F. Brown et al., Solid State Commun. 9, 487 (1971).

[23] M. R. Thuler et al., Phys. Rev. B 27, 2082 (1983).

[24] G. van der Laan, J. Phys. Condens. Matter 3, 7443 (1991).

[25] R. Cowan, The Theory of Atomic Structure and Spectra (University of California Press, Berkeley, 1981).

[26] S. Sugano et al., Multiplets of Transition Metal Ions in Crystals (Academic Press, New York and London, 1970).

[27] J. Sakurai, Advanced Quantum Mechanics (AddisonWesley, Reading, MA, 1967).

[28] O. Keski-Rahkonen and M. O. Krause, At. Data Nucl. Data Tables 14, 139 (1974). 